\title{
Multiple on-line data collection and processing for radioimmunoassay using a micro-computer system
}

\author{
N WYNNE CARTER, D DAVIDSON, DF LUCAS, AND PD GRIFFITHS \\ From the Faculty of Medicine Computing Unit, University of Dundee, and Department of Biochemical \\ Medicine, Ninewells Hospital and Medical School, Dundee DDI 9SY, UK
}

SUMMARY A micro-computer system is described which has been designed to perform on-line data capture from up to seven radioisotope counters of different types in parallel with interactive results processing and subsequent transmission to a laboratory computer-based data management system.

With the purchase of a new radioactivity counter for use with radioimmunoassay (RIA), a Nuclear Enterprises 1600 (NE1600), resources were available to purchase a dedicated programmable calculator (Hewlett Packard). The data output from this would have to be entered into the laboratory's data management system based on a Modular One/8000 Computer (Computer Technology Ltd). ${ }^{12}$ The data from the existing counters have been output on paper tape produced by a teleprinter attached to each counter. This method has some drawbacks: long lengths of paper tape are liable to tear, and heavy use of the teleprinter's punches leads to wear and tape errors. Since, for a little extra expenditure, it was possible to develop a more flexible system which would handle the capture and processing of data not only from the new but from all the laboratory's counters, it was decided not to purchase the calculator. The choice lay between connecting the counters directly on-line to the main computer, like other instrumentation, or developing a self-contained system which would be on-line to the main computer. The latter is preferable because some of the counting is done over long periods such as overnight and at weekends. Furthermore, the stored data can be accepted by the main computer at low load times, and such a development is in keeping with the trend for most new analytical equipment to contain its own local primary processing capability.

\section{Hardware}

A micro-computer was assembled from the Intel SBC (single board computer) (Fig. 1) range of

Received for publication 30 October 1979 modules housed in an Intel System 80/20 chassis which provides a card cage, bus, and power supply. A cabinet was used to package the chassis together with a Periflex twin double-density, floppy disc drive (Fig. 2). Total cost was about $£ 6000$.

Figure 3 shows the positions of each of the system modules:

(a) an SBC 80/20/4 (Fig. 1) processor board with 4k bytes of RAM (random access memory), $4 \mathrm{k}$ bytes of EPROM (erasable programmable read only memory) timers, interrupt processors, V24 controller (CCITT recommendations V23 and V24) used to drive a 30 cps keyboard printer, etc.

(b) two SBC boards which provide the floppy disc interface

(c) one board (SBC 016) with 16k bytes of RAM

(d) one board (SBC 310) to provide hardware floating point arithmetic

(e) two boards (SBC 534), each providing four V24 interfaces (one for each counter and one for connection to the CTL Modular One).

The only local fabrication necessary was the provision of small configurable interface units (details available from authors) to connect to the existing counters, such as LKB Wallac, NE8000 series, etc., which use the $20 \mathrm{~mA}$ convention for driving their teletypes. The NE1600 is available with a V24 output option. These interface units also enable the teleprinter output to be retained if still desired.

\section{Software}

Available with the Intel processor was a simple software 'monitor' program which is plugged into two of the EPROM positions. Unfortunately, it is not designed to support the concept of interrupt- 
Fig. 1 Intel SBC 80/20/4. A typical single board computer module.

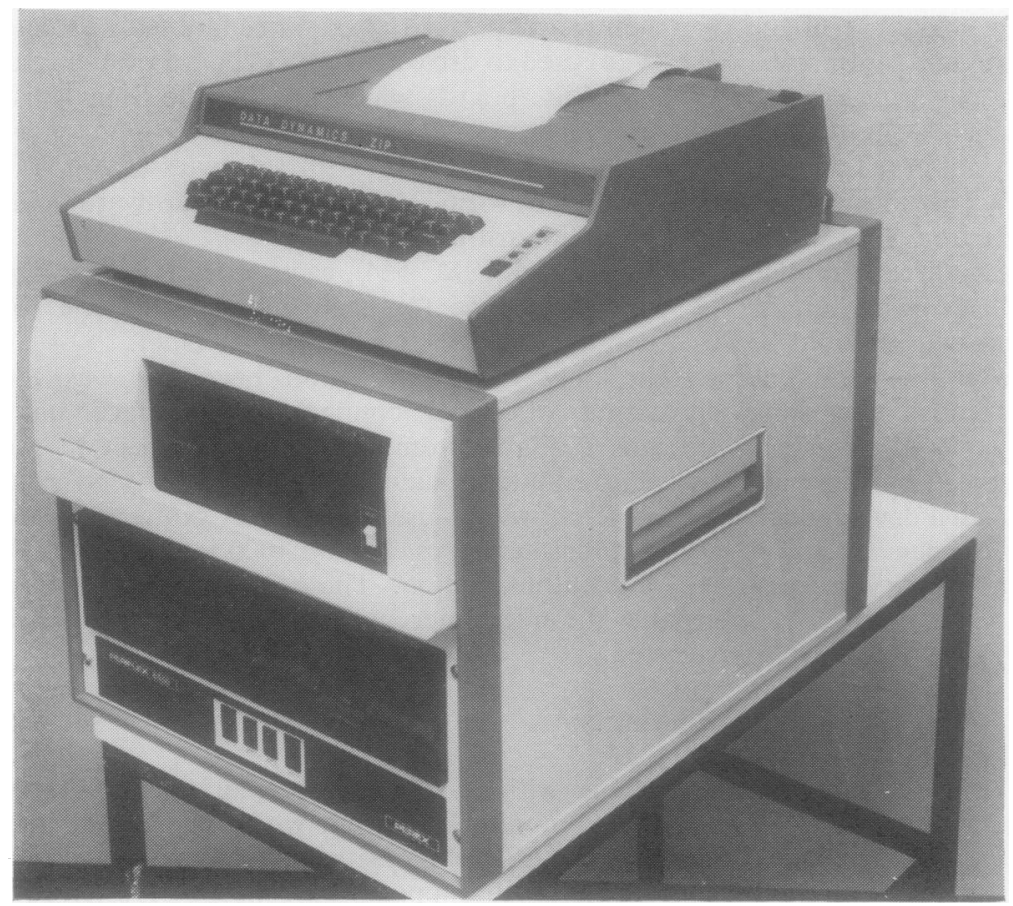

Fig. 2 Complete RIA micro-computer system. 


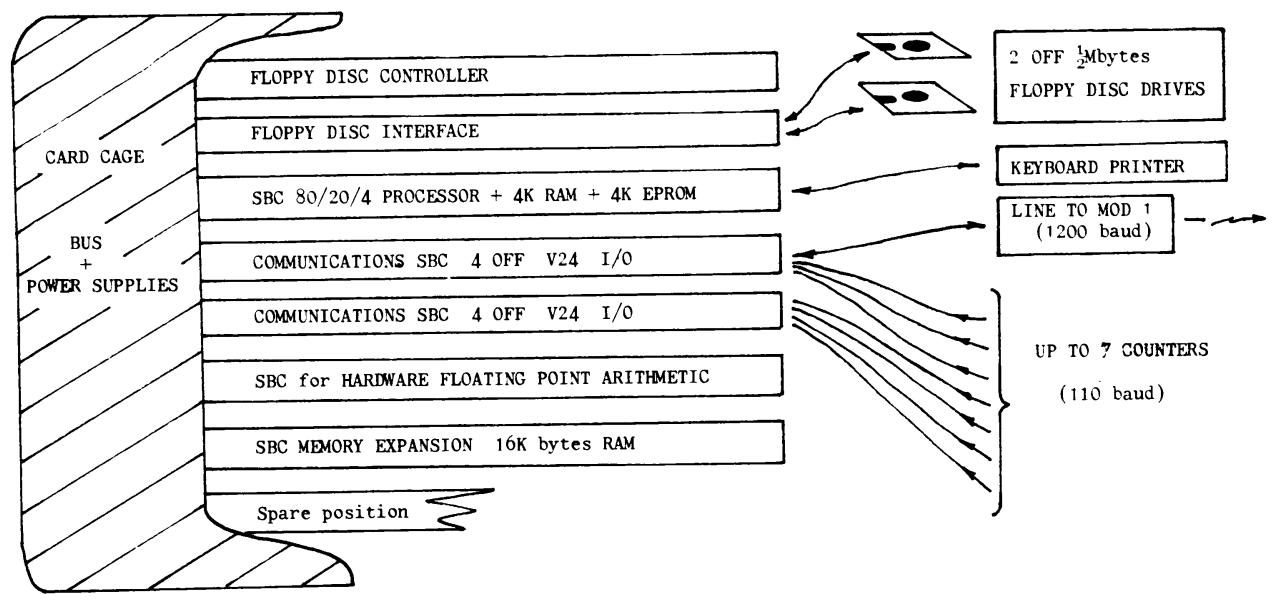

Fig. 3 Hardware configuration - Intel 8080 based micro-computer

driven data input from the counters at the same time as interrupt-driven disc transfer necessary for this application. As a result, a more appropriate suite of code, $4 \mathrm{k}$ bytes in size, was written to act as a 'supervisor' and 'operating system' which could be loaded from disc. The supervisor provides system initialisation, multilevel disc transfer, input/output routines (mimicking the main computer's user interface), and other basic facilities, and the operating system controls the loading, cataloguing, and selective running of a suite of application programs also held on disc. Another $4 \mathrm{k}$ bytes are consumed by the code, parameter tables (which hold status and control bytes together with counter data output format for each counter), and data buffers used for the on-line data input. This section is loaded from disc at startup and remains resident. The remaining $8 \mathrm{k}$ bytes accommodate one of the catalogued programs at a time as required.

The software was developed on a Modular One and 'down-line' loaded into the Intel microcomputer via the V24 communications line. Once established on a floppy disc the micro-system is self-contained and can be replicated using a disc-todisc copy program. (A disc formatting program, which uses the random sector addressing option, provides a significant performance improvement over the sequential format used by the Intel monitor software.)

To simplify system start-up, a bootstrap loader (a short program entered from the Intel monitor) reads the systems software into store from disc and transfers control to the operating system con- versation. This loader is now fixed into an EPROM chip which fits into one of the remaining processor board positions. Table 1 summarises the store utilisation.

For ease of use, the whole system operates using only one of the floppy disc drives, reserving the other for copying and fall-back in the event of one failing. Each floppy disc provides $512 \mathrm{k}$ bytes of data storage and, apart from the sections used for systems and logging software and program catalogue mentioned above, a further area is used as a catalogue of parameter tables, one for each type of radioimmunoassay method used. A user-driven catalogued program 'ESTC' permits a conversational establishment of these tables. Table 2 shows one of these estimation tables.

The remaining disc area is used for data buffers. Both the sharing of counter runs by different estimations and the need for each sample to be run in duplicate, or higher replication frequencies, prohibit a one-to-one relationship between 'rawdata' input and the subsequent processed results. To provide the greatest flexibility, the disc area is divided into 'raw' and 'processed' batch buffers, and these are interleaved to minimise head movement, which, inevitably, is slow with the simple wormdriven mechanism that is employed. Figure 4 summarises the disc utilisation.

Catalogued programs are available to initiate counting runs into raw data batches (labelled A, B, $\mathrm{C}$, etc), to list and edit both raw and processed batches (the latter labelled $11,12,13$, etc. to avoid confusion with the main computer system batch 
Table 1 Store utilisation for radioimmunoassay

\begin{tabular}{|c|c|c|}
\hline & & HEX Address \\
\hline INTEL MONITOR in 'EPROM' & $1 \mathrm{~K}$ bytes & $\begin{array}{l}0000 \mathrm{H} \\
07 \mathrm{FFH}\end{array}$ \\
\hline BOOTSTRAP in 'EPROM' & $1 \mathrm{~K}$ bytes & $\begin{array}{l}0800 \mathrm{H} \\
11 \mathrm{FFH}\end{array}$ \\
\hline Unused 'EPROM' position & $1 \mathrm{~K}$ bytes & $\begin{array}{l}1200 \mathrm{H} \\
15 \mathrm{FFH}\end{array}$ \\
\hline Address Gap & & \\
\hline SBC 80/20/4 'RAM' (Stack etc.) & $4 \mathrm{~K}$ bytes & $\begin{array}{l}3000 \mathrm{H} \\
3 \mathrm{FFFH}\end{array}$ \\
\hline OPERATING SYSTEM & $4 \mathrm{~K}$ bytes & $\begin{array}{l}4000 \mathrm{H} \\
4 \mathrm{FFFH}\end{array}$ \\
\hline Store-resident COUNTER SCANNER : TABLES & $4 \mathrm{~K}$ bytes & $\begin{array}{l}5000 \mathrm{H} \\
5 \mathrm{FFFH}\end{array}$ \\
\hline $\begin{array}{l}\text { RE- SABLE AREA FOR ONE BACKGROUND } \\
\text { PROGRAM AT A TIME }\end{array}$ & $8 \mathrm{~K}$ bytes & $\begin{array}{l}6000 \mathrm{H} \\
7 \mathrm{FFFH}\end{array}$ \\
\hline
\end{tabular}

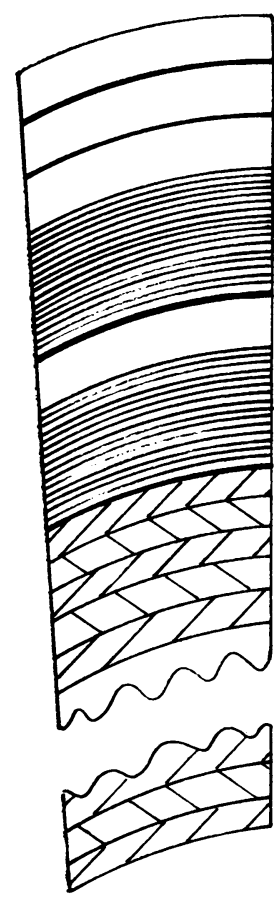

\section{DISC COPY OF OPERATING SYSTEM \\ CATALOGUE $\overline{O F ~ B A C K G R O U N D}$ TASK NAMES \\ '20' BACKGROUND TASK PROGRAMS}

DISC COPY OF STORE-RESIDENT CODE and TABLES

CATALOGUE OF ESTIMATION CONTROL PARAMETERS

'25' ESTIMATION CONTROL PARAMETER TABLES

'25' RAW and '25' PROCESSED DATA BATCHES

[ Interleaved sectors for 'Raw' and 'Processed' batches to reduce head movement in processing from

'Raw' to 'Processed'. ]
Fig. 4 Floppy disc utilization for radioimmunoassay 
Table 2 An estimation parameter table

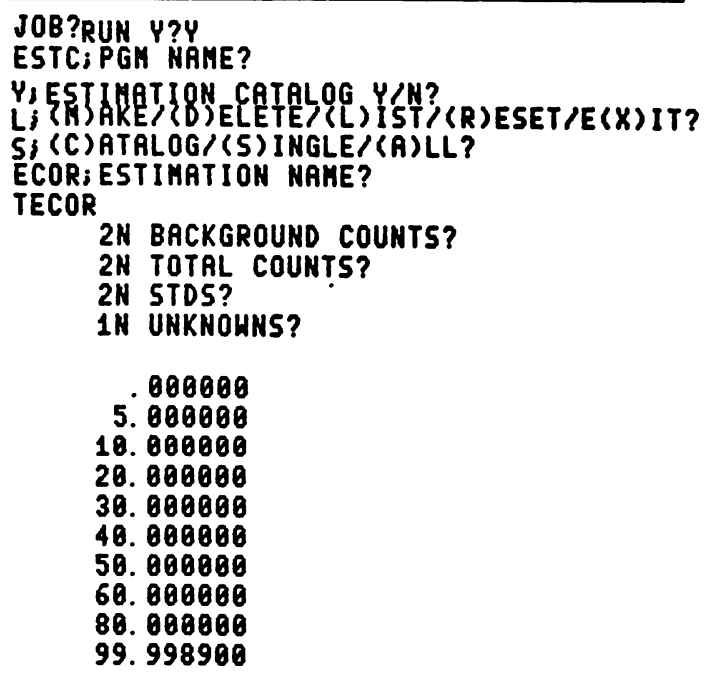

MOD I SCALE FACTOR? 2

\section{CONC}

MINIMUN YALUE $\quad .000$

YALUE OF INCREMENT 2.500

HO. OF PLACES AFTER DEC. POINT

function, places its results into a nominated processed batch, although source data can be taken from selected sections of the raw counter batches to permit mixed counting. After the total, background, and standard counts have been processed, three calibration graphs can be plotted on the printer (CØ/CB-1, COUNTS, and PER CENT. BOUND versus CONCENTRATION) and the most appropriate chosen for calibration. Processing can be repeated after editing of raw data if required. The results for the subsequent patient samples are then computed by linear interpolation between adjacent standards, with measures of precision, and placed in the chosen processed batch on disc. The appropriate estimation parameter table specifies the batch format, such as replication frequencies, for each of total and background counts, standards and samples, as well as the number of and assigned values for the standards. The graph dimensions are also specified using 'ESTC'. Table 3 illustrates a selection of printouts and Fig. 5 a typical graph.

Finally, when it is convenient, the catalogued program 'XMIT' is used to transfer a processed batch into the Modular One. The input software in the Modular One uses a small module specific to this device but shares with other on-line instruments the remaining data acquisition software.

It may be valuable also to note some of the data

CO/CB-1

MINIHUN YALUE

YALUE OF INCREHENT 180

NO. OF PLACES AFTER DEC. POINT 3

COUNTS/TIKE

MINIHUN YALUE

15. 008

YALUE OF INCREHENT 1.250

NO. OF PLACES AFTER DEC. POINT

\section{$\%$ BOUND}

MINIHUH YaLUE

YALUE OF. INCREMENT

10. 000

1. 008

NO. OF PLACES AFTER DEC. POINT

\section{Y $($ (H) AKE/(D)ELETE/(L)IST/ (R)ESET/E (X)IT?} JOB?

buffer numbers), and also to load and edit laboratory accession numbers and quality control identifiers in the processed batches. The catalogued program 'PROC', which is called in to perform the processing 
Table 3 A selection of printouts (a) Raw data batch buffer; (b) Part of the output from 'PROC'; (c) A processed batch ready for transmission to the Modular One

\begin{tabular}{ccrrr} 
(a) CUP & TRŖY & TUBE & TIME & \multicolumn{1}{c}{ COUNT } \\
1 & TI & 1 & 66 & 9 \\
3 & $\mathrm{~J}$ & 2 & 60 & 6 \\
4 & $\mathrm{~J}$ & 3 & 60 & 10618 \\
5 & $\mathrm{~J}$ & 4 & 68 & 9981 \\
5 & $\mathrm{~J}$ & 5 & 60 & 5284 \\
6 & $\mathrm{~J}$ & 6 & 68 & 5270 \\
7 & $\mathrm{~J}$ & 7 & 68 & 4231 \\
8 & $\mathrm{~J}$ & 8 & 68 & 4398 \\
9 & $\mathrm{~J}$ & 9 & 68 & 3738 \\
18 & $\mathrm{~J}$ & 16 & 66 & 3873 \\
11 & $\mathrm{~J}$ & 11 & 68 & 2829 \\
12 & $\mathrm{~J}$ & 12 & 68 & 2996 \\
13 & $\mathrm{~J}$ & 13 & 68 & 2366 \\
14 & $\mathrm{~J}$ & 14 & 68 & 2382 \\
15 & $\mathrm{~J}$ & 15 & 68 & 1984 \\
16 & $\mathrm{~J}$ & 16 & 68 & 1975
\end{tabular}

(b) RCUP TIME COUNT CT/TIME PCBND CO/CB-1 RESULT RUALITY

\begin{tabular}{|c|c|c|c|c|c|c|c|c|}
\hline $\begin{array}{l}1 \\
2 \\
3 \\
4\end{array}$ & $\begin{array}{l}60 \\
60 \\
60 \\
60\end{array}$ & $\begin{array}{r}9 \\
6 \\
10618 \\
9981\end{array}$ & $\begin{array}{l}\text { BKG } \\
\text { T.CN } \\
1725 \\
171.658\end{array}$ & $\begin{array}{l}\text { EC } \\
\text { QC }\end{array}$ & & & & \\
\hline $\begin{array}{l}5 \\
6\end{array}$ & $\begin{array}{l}60 \\
68\end{array}$ & $\begin{array}{l}5204 \\
5270\end{array}$ & $\begin{array}{l}\text { STD } \\
87.283\end{array}$ & 58.811 & .800 & .080 & SC & .645 \\
\hline $\begin{array}{l}? \\
8\end{array}$ & $\begin{array}{l}60 \\
60\end{array}$ & $\begin{array}{l}4231 \\
4398\end{array}$ & $\begin{array}{l}\text { STD } \\
71.842\end{array}$ & 41.889 & .215 & 5.680 & SC & 1. 712 \\
\hline $\begin{array}{r}9 \\
18\end{array}$ & $\begin{array}{l}68 \\
68\end{array}$ & $\begin{array}{l}3730 \\
3873\end{array}$ & $\begin{array}{l}5 T 0 \\
63.358\end{array}$ & 36.864 & .378 & 18. 808 & BC & 1. 648 \\
\hline $\begin{array}{l}11 \\
12\end{array}$ & $\begin{array}{l}68 \\
68\end{array}$ & $\begin{array}{l}2829 \\
2996\end{array}$ & $\begin{array}{l}\text { STD } \\
48.542\end{array}$ & 28. 226 & .800 & 28. 680 & BC & 2. 188 \\
\hline
\end{tabular}

(c) CUP LABNO RESULT

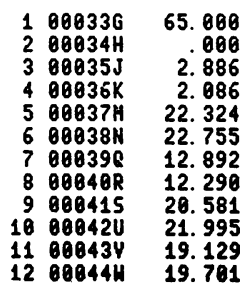

storage and processing constraints. 'Raw' data is stored in the form:

TIME-16 bit integer, COUNT-16 bit integer.

Processing is performed using the hardware floating point unit which has an 8 bit exponent and a 23 bit mantissa, and all derived values are stored in this form as four bytes.

The quality monitor is based on removing replicate counts which are more than $3.6 \mathrm{SD}$, from the mean, and repeating this process until too few remain or an acceptable dispersion is found.

The final results are therefore constrained only by the initial data. Much has been written discussing various curve-fitting techniques, but there is considerable agreement ${ }^{3}$ that the greater flexibility to accommodate differing curve shapes offered by linear interpolation makes it the preferred choice for a general-purpose facility. Careful attention to positioning and frequency of calibration standards across the working range can be used to minimise calculation error. It was also felt that the display of the various calibration options in graphical form, before processing, would offer a visual judgement of suitability to be made $^{3}$ and if it is considered appropriate, the editing facilities can be used to adjust the raw data values. Indeed, this entire cycle of operation can be repeated as often as necessary with no loss of source data, which helps to reduce the 
number of occasions on which a complete re-assay of a batch of specimens is necessary.

\section{Discussion}

The micro-computer was assembled to meet the exact requirements of the task. No hardware expertise was necessary, and a convenient commercially available SBC micro-technology packaging system was used. The initial investment of approximately four person-months in providing development aids and systems software will serve also for other future applications. The programs specific to this particular application have taken only three personmonths to produce and are very simple in nature. Their division into separate catalogued modules will permit modifications to be made readily and extra functions to be developed as the need arises. The day-to-day operation and configuration necessary to add extra estimations and modify existing ones can be performed by laboratory technical staff. This development is consistent with the desirable concept of distributing the immediate data processing from analytical instrumentation and has become financially possible with the advent of micro-technology. Indeed the system described has been designed to operate either in a free standing mode or on-line to any laboratory data processing system which supports V24 devices such as VDUs.
Since the hardware is available in off-the-shelf form from a number of suppliers, and the entire software package can be transported on a standard floppy disc, it could easily be installed in other laboratories. Full documentation which covers support and operation has been prepared.

\section{References}

${ }^{1}$ Carter NW. Development of a Computer System, based on Modular One, for Pathology Laboratories. In: Conference on Computers for Analysis and Control in Medical and Biological Research, University of Sheffield, 1971 (IEE Conference Publications, 79). London: IEE.

${ }^{2}$ Carter NW, Griffiths PD, White CJ, Chow, MC, Lucas DF. Design and implementation of a real-time computer system for diagnostic medical laboratories. In: Anderson J and Forsythe JM, eds. Medinfo 74: Proceedings of the 1st World Conference on Medical Informatics, Stockholm, 1974. Amsterdam: NorthHolland; New York: American Elsevier, 1975: 975-8.

${ }^{3}$ Vogt W, Sandel P, Langfelder C, Knedel M. Performance of various mathematical methods for computer-aided processing of radioimmunoassay results. Clin Chem Acta 1978; 87:101-11.

Requests for reprints to: Dr N W Carter, Faculty of Medicine Computing Unit, Ninewells Hospital and Medical School, PO Box 120, Dundee, DD1 9SY, UK. 